\title{
Evaluation of Cervical lymphnode Metastasis in Oral Squamous Cell Carcinoma: Comparison between Palpation, USG, CT Scan \& Histopathology
}

\author{
Dr. Aniket Kansara ${ }^{1}$, Dr S.M.Sharma ${ }^{2}$ \\ ${ }^{l}$ Consultant Maxillofacial Surgeon, Ahmedabad \\ ${ }^{2}$ Dept of Oral \& Maxillofacial Surgery, A.B.Shetty Memorial Institute of Dental Sciences, Mangalore
}

\begin{abstract}
:
Background:The presence or absence of nodal metastasis has a great impact on the prognosis and survival of patients with head and neck cancer. The risk of occult metastasis is related to the method by which the lymph nodes are evaluated. It is possible to reduce the risk of undiagnosed metastasis with accurate imaging techniques and thus probably reduce the number of elective neck treatments.

Aims and objectives:To assess the accuracy of clinical palpation, CT Scan and Ultrasound in prediction of lymph node metastasis in oral squamous cell carcinoma so that a suitable surgical neck dissection can be carried out.

Methods :Twenty patients with oral squamous cell carcinoma who underwent 20 neck dissections (15MRND, 5 SOHND) were included. All the patients underwent examination of neck pre operatively by palpation, Computed Tomography with contrast and Ultrasound for node detection. The findings were correlated with the results of histopathologic examination of the neck specimen. The results were obtained after statistical analysis.

Results:Ten neck dissection specimens showed metastatic lymph node involvement in postoperative histopathology. Lymph node involvement was identified preoperatively by palpation in 8 necks, CT in 6necks and US in 17 necks. The palpation showed 80\% sensitivity, 30\% specificity. CT showed sensitivity of 50\%, specificity of $90 \%$ and US showed sensitivity of $90 \%$, specificity of $20 \%$.

Conclusion:We recommend that the use of preoperative US and CT scanning of the neck by an experienced radiologist is essential and useful for diagnosis, staging and therapy choices which will help to reduce patient morbidity.
\end{abstract}

Keywords: Oral squamous cell carcinoma $\cdot$ Occult metastasis $\cdot$ Computed

tomography· Ultrasound

\section{Introduction}

Oral cancer is a major cause of morbidity worldwide; ninety percent of oral malignancies are squamous cell carcinomas. ${ }^{1}$ Oral cancer is an increasingly prevalent disease that accounts for the demise and deformity of many patients. The incidence of oral cancer, its anatomical complexity, esthetic repercussions and its utility in the basic functions of mastication, deglutition and speech make it in many ways the most challenging head and neck tumor management.

Early detection would drastically bring down the morbidity and mortality associated with advanced stages of oral cancer. In the treatment of patients with squamous cell carcinoma of the oral cavity size of primary lesion, site, thickness, histological staging and the presence or absence of metastatic spread are all important to formulate a treatment plan. ${ }^{2}$

Invasive squamous cell carcinoma of the upper aero-digestive tract has a strong potential for metastatic spread to the cervical lymph nodes. The presence or absence of nodal metastasis has a great impact on the prognosis and survival of patients with oral cancer. Nodal metastasis to one side decreases the survival by $50 \%$, while bilateral metastasis decreases survival by a further $25 \% .{ }^{2,3,4,5} \mathrm{Hence}$, the neck status is the single most important indicator of prognosis in head and neck cancers. ${ }^{6}$

Factors that have been shown to increase the risk of cervical metastasis include the site of the primary tumor, tumor thickness, DNAploidy and tumor growth patterns such as infiltrating margins, perineural spread and angioinvasion. Despite this knowledge it remains difficult to accurately predict the metastatic behavior of these lesions. ${ }^{7}$

Lymphatic metastasis is a frequent event that influences prognosis, a decision to treat the cervical lymph nodes has to be made in almost all patients, even if metastasis is not apparent clinically. It is therefore important to assess regional nodal involvement as reliably as possible.

Management of the neck in patients with oral SCC is controversial. Some clinicians and surgeons advocate elective treatment of the neck while others don't. ${ }^{2}$ The risk of occult metastasis that is higher than $20 \%$ 
is the most important indication for elective neck treatment. It is possible to reduce the risk of undiagnosed metastasis with accurate imaging techniques and thus probably reduce the number of elective neck treatments. ${ }^{8}$

Assessment for the presence of cervical lymph nodes is traditionally carried out by palpation; however palpation may be difficult in operated patients, after radiation therapy and in patients with short bulky necks. It is also important to assess carotid arterial invasion by lymph nodes. Imaging studies are better than clinical evaluation in predicting carotid artery invasion. Computed Tomography and Ultrasonography are the modalities which are most commonly used for this purpose. ${ }^{10}$ In recent years Positron Emission Tomography with 2- fluoro2-deoxy-d-glucose (F-FDG) has become an additional tool for the staging. ${ }^{11}$

Technological advances in radiology over the last decade have made Computed tomography and Magnetic resonance imaging the primary tools for evaluating head and neck cancer. The acid test for an imaging method is how well it fares in the clinically No neck.CT has good sensitivity and specificity for the evaluation of lymph node metastasis, MRI is sensitive but not specific. Though MRI is useful, is expensive and not always available. In addition differentiation between nodes with and those without metastasis on the radiologic characteristics has low specificity and it remains difficult and unreliable.Ultrasound can detect a high number of nonpalpable enlarged lymph nodes, but when sensitivity to detect metastases increases, the specificity inevitably decreases.

So the purpose of this prospective study is to compare the diagnostic efficacy of clinical palpation, CT Scan, Ultrasonography in detection of metastatic lymph nodes in oral squamous cell carcinoma by correlating these results with post operative histopathology after neck dissection so that an evidence based protocol can be evolved for management of oral squamous cell carcinoma.

\section{Material \& method}

20 patients with squamous cell carcinoma of oral cavity requiring surgical treatmentin Department of Oral \& Maxillofacial Surgery ,A.B.Shetty Memorial Institute Of Dental Sciences, Mangalore were included. Patients who have undergone radiotherapy and chemotherapy and patients with inoperable disease that is extensive primary growth or the presence of distant metastasis, any systemic condition contraindicating General Anaesthesia were excluded. The site of primary tumor was lip in 4(20\%) patients ,Retromolartrigone in $2(10 \%)$ patients, Buccal Mucosa in 3(15\%) patients, Lower Alveolus in 8(40\%) patients, Tongue in 2(10\%) patients and Floor of Mouth in 1(5\%) patient. All the patients were examined clinically and then examination of neck was carried out by CT and US. Sensitivity, specificity, positive predictive value, negative predictive value and accuracy were calculated by using the histopathologic findings in the neck dissection specimen as a standard of reference. Findings at these modalities and histopathologic examination were compared for the overall number of nodes in the neck.

\section{Palpation:}

The criteria to consider node as metastatic on palpation was a firm to hard fixed node more than $10 \mathrm{~mm}$ in size.

\section{Computed Tomography:}

All the patients were examined by CT of neck from the base of the skull to the clavicle. Axial CT sections were taken on a GE Bright Speed 16 slice spiral CT machine with $5 \mathrm{~mm}$ sections contiguously. $40-50 \mathrm{ml}$ of non ionic contrast material was administered intravenously in all patients.

The criteria used to define a node as metastatic in our study are: ${ }^{13}$

1. Lymphnodes greater than $1.5 \mathrm{~cm}$ in size

2 Nodes with central necrosis, regardless of size in the absence of clinical infection

3 Aggregation of nodes regardless of size

4 Evidence of extracapsular spread

5 Evidence of vascular invasion seen on contrast CT

\section{Ultrasonography:}

Ultrasonographic examination of the neck on both the sides was carried out using GE Voluson (730

Expert Series) USG machine and high frequency $(7 \mathrm{MHz}-14 \mathrm{MHz})$ linear transducer.

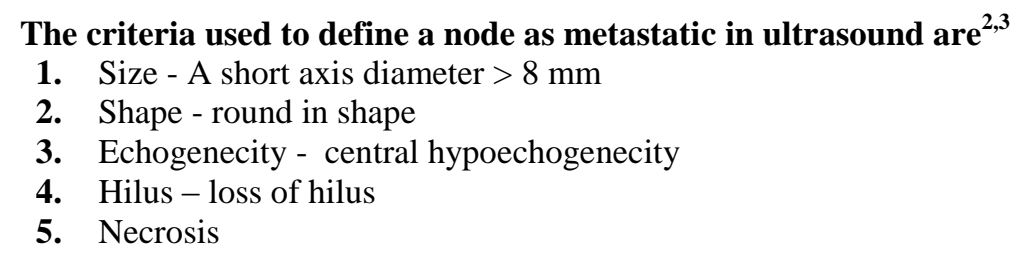


6. Extracapsular spread - irregular margin

7. Roundness index $-<2$ were considered malignant

All the patients underwent unilateral neck dissection. Supraomohyoid (SOHND) neck dissection was done in N0 neck and Modified Radical neck dissection (MRND) was done in N+ neck. In our study SOHND was done in 5 patients and MRND was done in 15 patients. Mandible was addressed by hemimandibulectomy and reconstruction was done with Pectoralis major myocutaneous flap.

Immediately after resection the specimen was spread out and labeled as level I- V according to the Memorial Sloan-Kettering classification and fixed in $10 \%$ formaldehyde solution. Subsequently neck dissection specimen was histologically examined as per institutional protocol and the findings of the pathologist were recorded per nodal level. The histological features of the lymph nodes were then compared with the pre operative CT and USG.

\section{Statistical Analysis:}

Diagnostic validity tests for different methods to assess the lymph node metastasis were performed. The results are presented in terms of sensitivity, specificity, predictive values and accuracy. Chisquare Test was used to assess the value of each screening method. A ' $\mathrm{P}$ ' value of 0.05 or less was considered for statistical significance.

True positive (TP) results are metastasis carrying (positive) nodes detected by any method True negative $(\mathrm{TN})$ results are unaffected by (negative) nodes demonstrated by any method False positive (FP) results are unaffected nodes demonstrated as carrying metastasis by any method False negative (FN) results are metastasis carrying nodes demonstrated as unaffected by any method Sensitivity determines how well positive lymph nodes are diagnosed by a particular method.

$$
\text { Sensitivity }=\frac{\text { True positive }}{\text { True positive }+ \text { False negative }}
$$

Specificity determines how well positive nodes are distinguished from non-affected nodes by a particular method

$$
\text { Specificity }=\frac{\text { True negative }}{\text { True negative }+ \text { False positive }}
$$

Accuracy determines how well a method functions

Accuracy $=\frac{\text { True positive }+ \text { True negative }}{\text { Total }} \quad$ X 100

Positive predictive value determines the probability of a node with a positive diagnostic result being actually positive

Positive predictive value $=\quad \frac{\text { True positive }}{\text { True positive }+ \text { False positive }} \quad$ X 100

Negative predictive value determines the probability of a node with a negative diagnostic result being actually unaffected

Negative predictive value $=$

True negative

True negative + False negative

X 100 


\section{Result}

Histopathological examination was considered gold standard for comparing these methods. 10 out of 20 neck dissection specimen contained metastatic deposits on histopathology. Out of 15 positive necks on palpation 8 had metastasis on histopathologic examination. 7 were false positive. Out of 5 negative necks on palpation 2 were positive on histopathologic examination giving false negative result.

17 necks were considered positive on US examination. Out of these 17 necks 9 were proved positive on histopathological examination. Out of 3 negative necks 1 had metastasis on histopathologic examination giving false negative result .

Out of 6 positive necks on CT scan 5 were positive on histopathological examination. 1 was false positive. Out of 14 negative cases on CT 5 had metastasis on histopathological examination giving false negative results in 5 cases.

Comparison between Palpation, US, CT with histopathology are shown in table 1.

The results of the statistical analysis are presented in table 2.

Using Chi-square test ' $\mathrm{P}$ ' value for palpation, US and CT is $0.102,0.018,0.197$. US is the only test showing statistical significant in our study.

Table 1: Comparison of Palpation, CT and USG with Histopathologic Examination

\begin{tabular}{|c|c|c|c|}
\hline \multirow{2}{*}{ Investigation } & \multirow{2}{*}{ Findings } & \multicolumn{2}{|c|}{ Histopathologic Examination } \\
\cline { 3 - 4 } & & Positive & Negative \\
\hline \multirow{2}{*}{ Palpation } & Positive & True Positive $(\mathrm{n}=8)$ & False Positive $(\mathrm{n}=7)$ \\
\cline { 2 - 4 } & Negative & False Negative $(\mathrm{n}=2)$ & True Negative $(\mathrm{n}=3)$ \\
\hline Computed & Positive & True Positive $(\mathrm{n}=5)$ & False Positive $(\mathrm{n}=1)$ \\
\cline { 2 - 4 } Tomography & Negative & False Negative $(\mathrm{n}=5)$ & True Negative $(\mathrm{n}=9)$ \\
\cline { 2 - 4 } & Positive & True Positive $(\mathrm{n}=9)$ & False Positive $(\mathrm{n}=8)$ \\
\hline \multirow{2}{*}{ Examination } & Negative & False Negative $(\mathrm{n}=1)$ & True Negative $(\mathrm{n}=2)$ \\
\cline { 2 - 4 } & & &
\end{tabular}

Table 2: Sensitivity, Specificity, Accuracy, Positive, Negative Predictive and 'P' Values of Methods Used

\begin{tabular}{|c|c|c|c|}
\hline Statistical Test & Palpation & Computed Tomography & $\begin{array}{c}\text { Utrasonographic } \\
\text { Examination }\end{array}$ \\
\hline Sensitivity (\%) & 80 & 50 & 90 \\
\hline Specificity (\%) & 30 & 90 & 52 \\
\hline $\begin{array}{c}\text { Positive Predictive } \\
\text { Value (\%) }\end{array}$ & 47 & 83 & 66 \\
\hline $\begin{array}{c}\text { Negative Predictive } \\
\text { Value (\%) }\end{array}$ & 60 & 64 & 55 \\
\hline Accuracy (\%) & 55 & & 0.018 \\
\hline 'P'Value * & 0.102 & 70 & \\
\hline
\end{tabular}

\section{Discussion}

Lymphatic metastasis is the most important mechanism in the spread of head and neck squamous cell carcinomas. The rate of metastasis probably reflects the aggressiveness of the primary tumor and is an important prognosticator. Not only the presence but also the number, level in the neck, size and the presence of extranodal spread are important prognostic features. ${ }^{19}$

Evaluation of cervical lymph nodes for malignancy has improved significantly in recent years through the introduction of modern imaging techniques such as US, CT, MRI and US-FNAC. Accurate radiologic imaging could potentially allow for a more conservative approach regarding management of the neck if the risk of occult metastatic disease could be reduced to $20 \%$. $^{20}$

Clinical palpation is the routine first line of method in evaluating metastatic cervical lymphadenopathy. In our study palpation results are comparable to various studies where they got sensitivity $79.5 \%$ \& $92.30 \%$ respectively. ${ }^{13,3}$ The sensitivity ranges from $0-92.3 \%$ in the literature reported. ${ }^{3,13,20}$ The high sensitivity of 
palpation is attributable to the use of only physical characteristics such as size and consistency. Even the reactive lymph nodes can also achieve the same dimensions as metastatic nodes and so false positive results are inevitable.The accuracy of $55 \%$ for palpation in our study can be comparable to previous studies ${ }^{13,17,18,22}$ but it ranged from $38.9-86 \%$ in the literature reported. $3,4,5,20,21$

US has got the highest sensitivity of $90 \%$ and lowest specificity of $20 \%$ in our study. This shows that our study has no false negative and more number of false positive results. The detection of more number of lymph nodes however inevitably leads to a lower specificity and as the differentiation between reactive and metastatic is based on morphologic criteria, this leads to low specificity. ${ }^{23}$ Our US results are comparable to previous studies. $4,17,18$ The results of our study shows that the accuracy of US alone never exceeds $70 \%$ as any rise in sensitivity is always accompanied by a decrease in specificity. ${ }^{12,14}$ In reported literature the accuracy of US ranges from $67-95 \%,{ }^{4,6,14,17}$ Advantages of US over other imaging techniques are it is economical, widely available and well tolerated by the patient, absence of radiation, lack of need for a contrast medium and ability to easy on-screen node measurements. It is the only available imaging technique that can be used for frequent routine follow up. It has disadvantages like primary tumor is seen infrequently and documentation of the finding is also a problem. $8,19,24$

CT has been used to determine neck metastasis in carcinoma of head and neck since 1981. Today there is some controversy about its usefulness. Criteria for assessing neck lymph nodes vary with many authors. M. August and M.Nguyen in 1994 proposed the radiologic criteria in CT for assessing cervical metastasis in patients with primary SCC of head and neck. Based on their study we used a size criteria $15 \mathrm{~mm}$, central necrosis , conglomeration, extracapsular spread and vascular invasion. Our CT results are comparable with previous studies. ${ }^{5,9,15}$

K.Saravanan et al in 2002 have got a sensitivity of $95.65 \%$, specificity of $66.65 \%$ and accuracy of $92.30 \% .^{3}$ In their study the conglomeration and central necrosis had a sensitivity and specificity of $100 \%$ thus increasing the accuracy of CT. But in our study none of the cases showed conglomeration or necrosis. So this indicates rim enhancement and necrosis are the highly specific indicators of metastasis. Though necrosis is a reliable criterion, it is unfortunately quite rare in small nodes. Although very small irregularities in contrast enhancement are present in many lymph nodes, it is very difficult to distinguish these small irregularities from artifacts or anatomic irregularities. So size of nodes plays an important role in assessing their nature. ${ }^{19}$ In our study accuracy of $70 \%$ for CT can be compared to previous studies. ${ }^{13,17,20}$ The accuracy ranges from $68 \%$ $92.30 \% .^{3,5,13,17,22}$

CT is capable of imaging the neck in any plane. Resolution and depiction of tissues in deeper planes is superior in CT. CT can show the primary tumor with its local extensions and documentation is possible. Disadvantages of CT are its cost, radiation and it can't be repeated every time. It is an invasive technique due to injection of contrast.

Continuous advance of techniques leads to increase in sensitivity of imaging modalities in the detection of lymph nodes. However differentiation between benign and metastatic nodes is not possible as far as ultrasound examination, CT and magnetic resonance imaging are concerned. Although several criteria have been developed for this purpose, specificity of all imaging modalities is low. ${ }^{18}$ In our study US is characterized by high sensitivity (90\%) when compared to palpation (80\%) and CT (50\%). The specificity of US is low (20\%) compared to palpation (30\%) and CT (90).

In the current study we compared the accuracy of palpation, CT and US in detecting cervical lymphnode metastasis in oral squamous cell carcinoma. CT has better overall accuracy than any other method (70\% versus $55 \%)$.

Though the specificity of US is lower than other methods it has highest sensitivity and negative predictive value. To reliably select patients who do not need elective treatment, criteria with a high negative predictive value should be chosen. To obtain this high negative predictive value, the number of false negative results should be as low as possible. ${ }^{16}$ So accordingly US has got the highest negative predictive value.

Normally in CT, axial or coronal sections are used. CT is a two dimensional reconstruction of the anatomy if a single scan picture is viewed. But US allows a multidirectional scanning by using different angulations of the transducer. This may be the reason why US showed a higher sensitivity than CT. This problem may be approached by using thin slices in CT investigation. ${ }^{9}$

The major advancement in the staging and management of oral cancer is the sentinel lymph node biopsy. This technique accurately stages the regional lymphatics based on the status of the first echelon nodes in the lymphatic basin draining the primary tumour site while limiting morbidity caused by unnecessary lymph node dissection. If this technique is proved to be more valid and practical it may replace the other investigational modalities in the assessment of neck metastasis. ${ }^{17}$ 


\section{Conclusion}

This study concluded that the sensitivity of US (90\%) and specificity of CT $(90 \%)$ was more in detection of cervical lymph node metastasis in oral squamous cell carcinoma. False positive and false negative results were there in all modalities of investigation. US findings were more correlated with pathologic findings than palpation, but CT gave the most effective and reliable results when it is combined with US in neck staging. However, it is questionable whether a diagnosis based on a combination of CT and US solves the problems concerning cervical lymph node metastasis because histological examination can show metastatic involvement in lymph nodes as small as $1 \mathrm{~mm}$, which cannot be detected by imaging. ${ }^{25}$

We recommend that the use of preoperative US and CT scanning of the neck by an experienced radiologist is essential and useful for diagnosis, staging and therapy choices which will help to reduce patient morbidity. In view of our small sample size further study is essential to establish the reliability of the criterion in a larger sample of cervical lymph nodes.

[1]. Jatin P. Shah, Cancer of the Head and Neck. 2001; 100

[2]. Hodder SC, Evans RM, Patton DW, Silvester KC. Ultrasound and fine needle aspiration cytology in the staging of neck lymph nodes in oral squamous cell carcinoma. British Journal of Oral and Maxillofacial Surgery 2000; 38:430-436.

[3]. Saravanan K, Bapuraj R, Sharma SC, Radotra BD, Khandelwal N, Suri S. Computed tomography and ultrasonographic evaluation of metastatic cervical lymph nodes with surgico-clinicopathologiccorrelation.The Journal of Laryngology and Otology March 2002; 116: $194-199$

[4]. D'Souza O, Hasan S, Chary G, Hoisala VR, Carrea M. Cervical lymph node metastases in head and neck malignancy-A clinical / ultrasonographic/histopathological comparative study. The Indian Journal of Otolaryngology: Head and Neck Surgery 2003; 55: 9093.

[5]. Haberal I, Celik H, Gocmen H, Akmansu H, Yoruk M, Ozeri C. Which is important in the evaluation of metastatic lympn nodes in head and neck cancer: Palpation, Ultrasonography or computed tomography? Otolaryngol Head Neck Surg 2004; 130: 197-201.

[6]. Som PM. Detection of metastasis in cervical lymph nodes: CT and MR criteria and differential diagnosis. AJR 1992; 158: 961-9.

[7]. Knappe M, Louw M, Gregor RT. Ultrasonography -Guided Fine -Needle Aspiration for the Assessment of Cervical Metastases. Arch Otolaryngol Head Neck Surg 2000;126:1091-1096

[8]. Atula TS, Grenman R, Varpula MJ, Kurki TJ, Klemi PJ. Palpation, Ultrasound and Ultrasound -Guided Fine Needle Aspiration Cytology in the assessment of cervical lymph node status in Head and Neck cancer patients. Head and Neck 1996; 18:545-551.

[9]. Jank S, Robatscher P, Emshoff R, Strobl H, Gojer G, Norer B. The diagnostic value of ultrasonography to detect occult lymph node involvement at different levels in patients with squamous cell carcinoma in the maxillofacial region. Int. J. Oral Maxilofac. Surg 2003; 32:39-42.

[10]. Shah JP, Patel SG. American Cancer Society Atlas of Clinical Oncology, Cancer of the Head and Neck. BC Decker Inc, Hamilton, London; 2001: pp 1.

[11]. Stuckensen T, Kovacs FA, Adams S, Baum RP. Staging of the neck in patients with oral cavity squamous cell carcinomas, a prospective comparison of PET, Ultrasound, CT and MRI. Journal of Cranio Maxillofacial Surgery 2000; 28:319-324.

[12]. Som PM, Curtin HD, Mancuso AA. Imaging -Based nodal classification for evaluation of neck metastatic adenopathy. AJR 2000; 174:837-845.

[13]. August M , Nguyen M . Evaluation of Metastatic Neck Disease by Computed Tomography . Int. J. Oral Maxillofac. Surg. 1994;23:290-293

[14]. Sonmez A, Ozturk N, Ersoy B , Bayramicli M, Celebiler O, Numanoglu A. Computed tomography in the management of cervical lymph node pathology. Journal of Plastic, Reconstructive \& Aesthetic Surgery. 2008;61:61-64

[15]. Shingaki S, Suzuki I, Nakajima T, Hayashi T, Nakayama H, Nakamura M. Computed tomographic evaluation of lymph node metastasis in head and neck carcinomas. Journal of CranioMaxillo-Facial Surgery 1995; 23:233-237.

[16]. Michiel WM, Brekel V, Stel HV, Castelijns JA, Nauta JP, Valk J et al. Cervical lymph node metastasis: Assessment of radiologic criteria. Radiology 1990; 177:379-384.

[17]. Sundar R, Rajesh P. Evaluation of clinical assessment, Ultrasonography and CT for detecting cervical lymph node metastasis in oral CA. Journal of Maxillofacial and Oral Surgery 2007;6:70-73.

[18]. Baatenburg RJ, Rongen RJ, Lameris JS, Harthoorn M, Verwoerd CD, Knegt P. Metastatic Neck Disease Palpation Vs Ultrasound Examination. Arch Otolaryngol Head Neck Surg 1989; 115:689-690.

[19]. Michiel WM, Brekel V, Castelijns JA, Snow GB. Diagnostic evaluation of the neck. Otolaryngologic clinics of North America 1998; 31:601-620.

[20]. Takes RP, Righi P, Meeuwis CA, Manni JJ, Knegt P, Marres HA et al. The value of ultrasound with ultrasound-guided fine needle aspiration biopsy compared to computed tomography in the detection of regional metastasis in the clinically negative neck. Int. J. Radiation Oncology Biol. Phys 1998; 40:1027-1032.

[21]. Michiel WM, Brekel V, Stel HV, Castelijns JA, Croll GJ, Snow GB. Lymph node Staging in Patients with clinically negative neck examinations by Ultrasound and Ultrasound -Guided Aspiraiton Cytology. The American Journal of Surgery 1991; 162:362366.

[22]. Righi PD, Kopecky KK, Caldemeyer KS, Ball VA, Weisberger EC, Radpour S. Comparison of Ultrasound- Fine Needle Aspiration and Computed Tomography in patients undergoing elective neck dissection. Head and Neck 1997; 19:604-610.

[23]. Takes RP, Knegt P, Manni JJ, Meeuwis CA, Marres HA, Spoelstra HA et al. Regional metastasis in Head and neck Squamous Cell Carcinoma: Revised Value Of US with US - guided FNAB. Radiology 1996; 198: 819-823.

[24]. Atula TS, Varpula MJ, Kurki TJ, Klemi PJ, Grenman R. Assessment of cervical lymph node status in head and neck cancer patients: palpation, computed tomography and low field magnetic resonance imaging compared with ultrasound -guided fine needle aspiration cytology. European Journal of Radiology 1997; 25:152-161.

[25]. Yusa $\mathrm{H}$, Yoshida $\mathrm{H}$, Ueno E. Ultrasonographic criteria for diagnosis of cervical lymph node metastasis of squamous cell carcinoma in the oral and maxillofacial region. J Oral MaxillofacSurg 1999;57:41-48 